\title{
Electrowetting display pixels fabricated by nanoimprint lithography
}

\author{
Q.T. Di ${ }^{1}$, H. Zhang ${ }^{2}$, X.L. Liang ${ }^{2}$, A.D. $\mathrm{Li}^{1}$, C.S. Xiao ${ }^{3}$ and D. $\mathrm{Wu}^{1 *}$, Q.Y. $\mathrm{Xu}^{4 \star}$ \\ 1.Materials Science and Engineering College of Engineering and Applied Sciences of Nanjing \\ University, Nanjing 210093, China
} 2.School of Electronics Engineering and Computer Science, Peking University,
Beijing100871,China

3. Wuhan University of Technology, Hubei 430070, China

4. Department of Physics, Southeast University, Nanjing 211189, China

Email: diwu@nju.edu.cn , xuqingyu@seu.edu.cn

Keywords: Electrowetting displays, nanoimprint lithography, RIE

\begin{abstract}
Electrowetting displays (EWD) is a novel technology, based on the variation in coverage of a colored oil film in pixels via application of an electrostatic force. These pixels are usually fabricated by photolithography, which seriously limits materials selection and large area fabrication. In this paper, we demonstrate the feasibility of a novel method, nanoimprint lithography (NIL), to fabricate EWD pixels. Controllable contraction and recovery of oil film in single EWD pixels was realized. Compared with conventional lithography, NIL provides a cost- and time- saving technique that generates comparable display properties and is able to be easily extended to the mass production.
\end{abstract}

\section{Introduction}

Hayes and Feenstra first reported the electrowetting displays (EWD) technology in 2003 [1], which employs an electrical control of wetting properties of liquid on dielectric surface. The voltage applied on the dielectric layer modifies the solid/liquid interfacial tension $\left(\gamma_{\mathrm{SL}}\right)$, which breaks the balance of interfacial tensions at the liquid, solid and vapor three-phase contacting point. The contacting angle $\beta$ changes with the applied voltage, following

$$
\cos \beta(\mathrm{V})=\cos \beta_{0}+\frac{\varepsilon}{2 \gamma_{L V} d} \mathrm{~V}^{2},
$$

where $\gamma_{\mathrm{LV}}$ is the liquid/vapor interfacial tension, $\varepsilon$ and $d$ are the dielectric constant and the thickness of the insulating layer, respectively, $\beta$ and $\beta_{0}$ are the contacting angles of the liquid drop on the dielectric surface in the presence and absence of voltage, respectively [2]. In a typical EWD pixel, as shown in Fig. 1, two kinds of liquids with opposite wetting characteristics (water and oil, for example) and an dielectric surface compose the three-phase system. The structure of an EWD pixel consists of a hydrophobic dielectric surface with hydrophilic grids. The colored oil drop is fully wetting on the dielectric surface, thus covers the whole pixel area in the absence of voltage, while the water is on the top of the oil layer, as shown in Fig. 1 (a). When a bias is applied on the dielectric layer, the oil drop will contract due to the decrease of the interfacial tension between water and dielectric layer. The pixels can be designed to work in reflective mode or transmission mode. The EWD is similar to the liquid crystal display (LCD), behaving as an optical switch. However, the energy consumption of EWD (effective transmission $>90 \%$ ) is much less than that of LCD (only <10\%) [3]. For the fabrication of large scale pixel arrays, photolithography was generally adopted, as described by Zhou et al. in 2009 [4]. Cytop and SU-8 photoresist were chosen as the hydrophobic dielectric and the hydrophilic grid, respectively, with the pixel dimension from $300 \times 900 \mu \mathrm{m}^{2}$ to $50 \times 150 \mu \mathrm{m}^{2}$. 


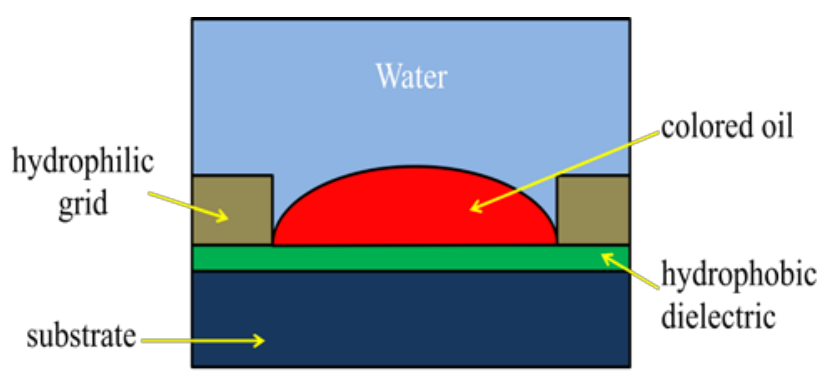

(a) OFF state

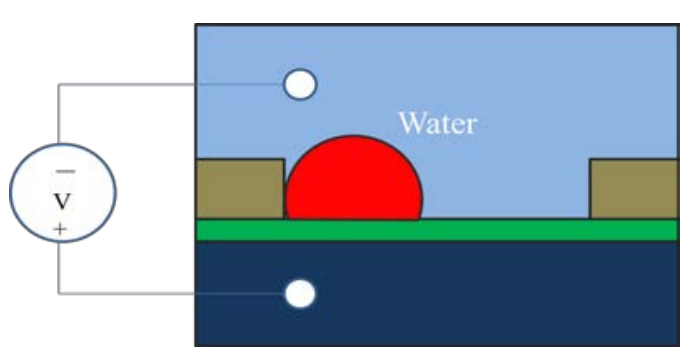

(b)ON state

Figure 1. Q. T. Di Diagram of electrical switching of an EWD pixel, schematic cross-section with (a) and without (b) voltage applied.

Nanoimprint lithography (NIL) is a recently developed novel process, in which a hard mold with surface relief pattern is pressed into a soft polymeric material to form a pattern. Obviously, the pattern in polymeric material is opposite to the mold. Therefore, the imprint resolutions are completely determined by the mold [5]. The imprint resolution improves with the improvement of the mold fabrication process. Additionally, the NIL process does not require complex optics and sophisticated light sources, hence are generally much less expensive than photolithography systems [6]. Due to these advantages, NIL is regarded as one of the most promising next generation lithography techniques. Various processes have been developed to fabricate nanoscale patterns by NIL, such as step and flash imprint lithography, soft lithography and thermal NIL [7]. The differences between these processes are primarily in the type of materials used for the mold and for the imprint resist. In this work, we use the thermal NIL process to fabricate the EWD pixels. As first reported in 1995, the thermal NIL process produces patterns by pressing a mold into a thermoplastic resist film as the film is heated above its glass transition temperature (Tg). The heated viscous resist flows and fills the mold with the application of a proper pressure. The mold is removed to leave the pattern in the film as the resist is cooled to solidify [5].

In this paper, we optimize thermal NIL parameters to fabricated EWD pixels. Display is demonstrated after filling the oil and sealing. It is shown that NIL might be an efficient alternative technique for the fabrication of EWD with significantly decreased fabrication cost, improved display quality. The results may help to promote the commercialization of EWD.

\section{Experimental Procedure}

The NIL fabrication procedure is shown in Fig. 2. Cytop and SU-8 photoresist are used as the hydrophobic dielectric and the hydrophilic grid materials, respectively. Cytop (Asahi, Cytop CTL $809 \mathrm{M}$ ), about $150 \mathrm{~nm}$ in thickness, was first spin-coated on a Si or an ITO substrate as the hydrophobic dielectric layer. $\mathrm{A} \mathrm{SiO}_{2}$ layer, 200-300 nm in thickness, was deposited on the Cytop layer using plasma enhanced chemical vapor deposition (PECVD) at $30{ }^{0} \mathrm{C}$ Then SU-8 (Gestate Snarl GM1050) was spin-coated on top of SiO2, as shown in Fig. 2(a). During the thermal NIL process, a $\mathrm{Si}$ mold is pressed into the SU-8 photoresist layer at $110{ }^{0} \mathrm{C}$. The temperature is maintained at $110{ }^{\circ} \mathrm{C}$ for 5 min with the application of a $0.3 \mathrm{MPa}$ pressure (Fig. 2 (b)), The applied pressure was released and the Si mold was removed after the temperature is below $40{ }^{0} \mathrm{C}$ (Fig. 2 (c)). O2/SF6 plasma was used in reactive ion-beam etching (RIE) to remove the SU-8 residual layer and the $\mathrm{SiO}_{2}$ removed by hydrofluoric acid to make the Cytop layer exposed, as shown in Fig. 2(d). Dodecane dissolved with red-164 dye was filled into the fabricated SU-8 grid, sealed under distilled water and tested as shown in Fig. 2(e). 


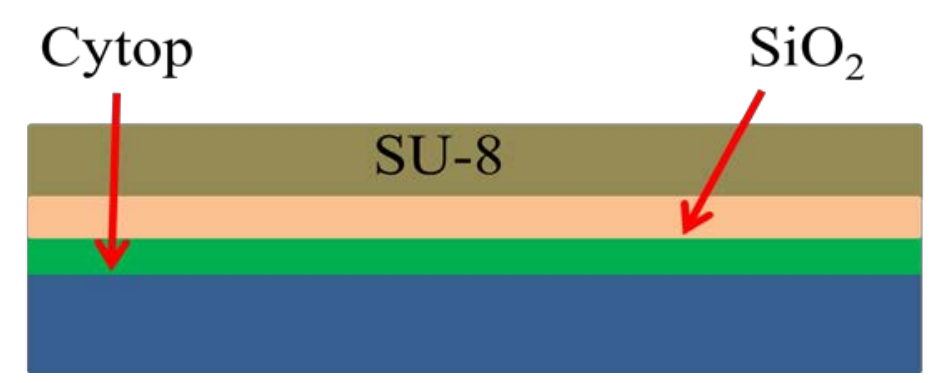

(a) the $\mathrm{SU}-8 / \mathrm{SiO}_{2} /$ Cytop multilayer

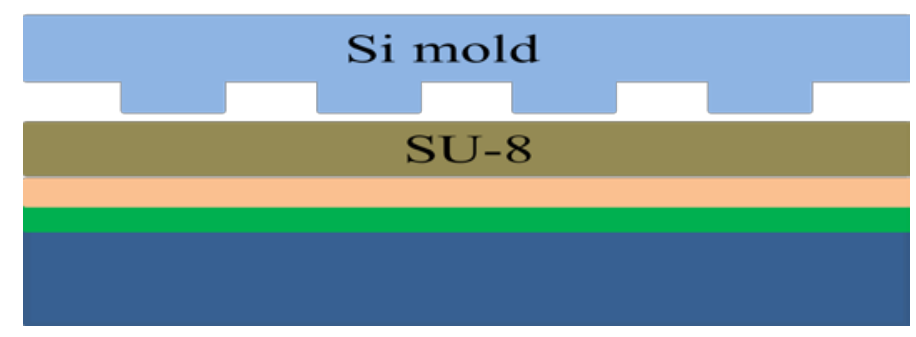

(b) pressing the mold into the resist during heating

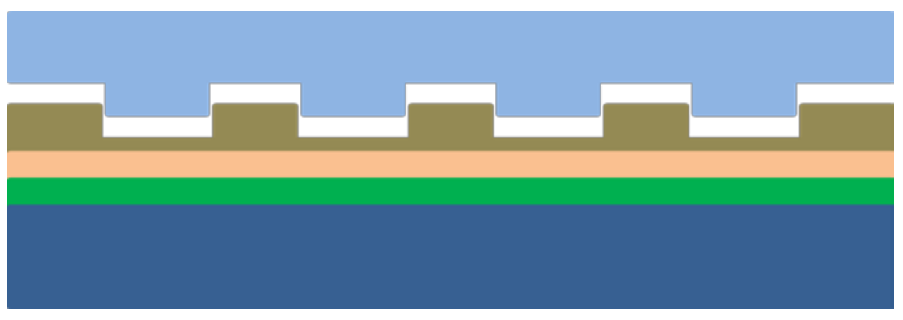

(c) mold removal, followed by curing the resist

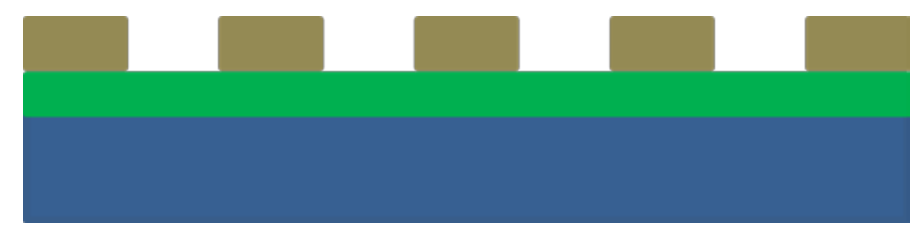

(d) removal the residue SU-8 layer by RIE and etching out the $\mathrm{SiO}_{2}$ by $\mathrm{HF}$

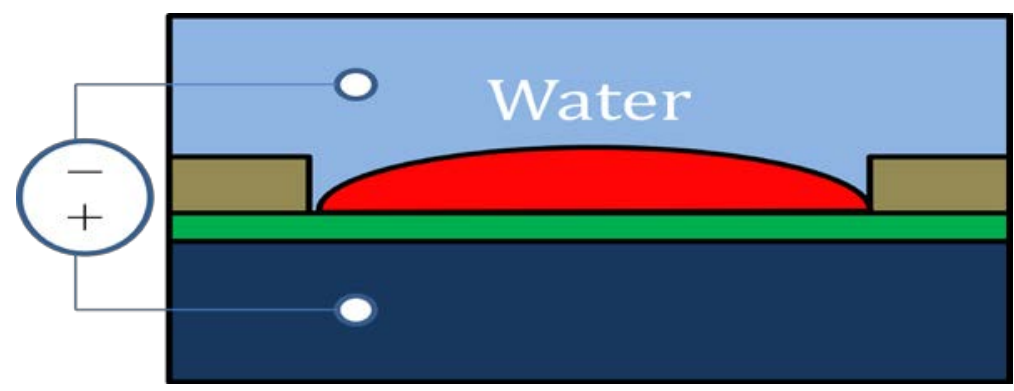

(e) filling the oil to test

Figure 2. Q. T. Di Schematics of EWD grids fabrication and test process.

\section{Results and discussion}

Cytop is an innovation of fluorpolymers and retains all the traditional merits of conventional fluorpolymers. SU-8 is an epoxy based, chemically amplified resist system with excellent sensitivity and high aspect ratios. Uncured SU-8 has advantages such as low glass transition temperature of $60{ }^{0} \mathrm{C}$ and good formability, which make it suitable for imprint lithography [8]. It is 
impossible to deposit SU-8 uniformly on Cytop because Cytop surface is hydrophobic while SU-8 is hydrophilic. Zhou et al. solved the problem by treating the Cytop surface with a low-power oxygen plasma to make Cytop hydrophilic. However an annealed at $180{ }^{\circ} \mathrm{C}$ for $30 \mathrm{~min}$ is required to recover the hydrophobic surface of Cytop [4]. This process is time-consuming and troublesome. In the current work, we deposit a thin $\mathrm{SiO}_{2}$ layer by PECVD on top of Cytop, as shown in Fig. 2 (a). SU-8 can then be uniformly spin-coated on the $\mathrm{SiO}_{2}$ layer with good adhesion since the $\mathrm{SiO}_{2}$ surface is hydrophilic.

A Si mold with rectangular column array was used to fabricate the grids. Demolding, which separates the mold from the resist, plays a critical role to the quality of resist replica. The friction and adhesion can cause deformation and breakdown of the transferred patterns on the resist [9]. Usually, an anti-sticking thin functional layer is applied on the mold to reduce the surface energy and suppress adhesion between the mold and the resist. Self-assembled monolayer of $\mathrm{CF}_{3}\left(\mathrm{CF}_{2}\right)_{7}\left(\mathrm{CH}_{2}\right)_{2} \mathrm{SiC}_{13}$ (FDTS), deposited on the oxidized Si mold surface, is used as the antiadhesive agent [10].

However, an anti-sticking layer on the Si mold is not enough to guarantee a successful imprinting of SU-8. The imprint procedures and parameters should be optimized. Previously, SU-8 is imprinted by thermal NIL and then cured by UV before demolding [11]. The difference in mechanical properties of various SU-8 resists make it difficult to achieve a fracture-free SU-8 grid in this way. Additionally, it may damage the expensive mold once the cured SU-8 sticks on it. We then propose that the demolding should be executed right after the thermal NIL process.

Since NIL is essentially a mechanical molding process, a residual layer always exists in the resist after imprinting. This residual layer should be removed by additional oxygen plasma etching process to complete the pattern transfer [12]. Although measures have been taken to attain uniform distribution of pressure over the entire mold surface, variations in the residual layer thickness still remain. This makes it difficult to remove the residual layer completely by RIE, because the Cytop layer underneath will be inevitably etched in areas where the residual SU-8 is thinner. In this way, the aforementioned $\mathrm{SiO}_{2}$ layer acts as a barrier to protect the Cytop layer. The cured SU-8 was etched in an $\mathrm{O}_{2} / \mathrm{SF}_{6}$ plasma, in which the small amount of $\mathrm{SF}_{6}$ was added to enhance the etching speed [13]. As measured, the etching speed of SU-8 is about $6-7 \mathrm{~nm} / \mathrm{s}$, while that of $\mathrm{SiO}_{2}$ is just around $1.5 \mathrm{~nm} / \mathrm{s}$ at same conditions. That is to say, the $\mathrm{SiO}_{2}$ layer should be thick enough to ensure that the residual layer can be removed completely and the Cytop layer is not damaged. Fig. 3 shows the cross-sectional SEM images of a fabricated grid as the SU-8 residual layer is removed by RIE. The $\mathrm{SiO}_{2}$ layer under the SU-8 grid is intact because that the thickness of the SU-8 grid is far larger than that of the residual layer (about $40 \mathrm{~nm}$ ). The observation of $\mathrm{SiO}_{2}$ layer between two adjacent SU-8 grids, as shown in Fig. 3(b), indicates the ability of $\mathrm{SiO}_{2}$ as a etching barrier. The thickness of $\mathrm{SiO}_{2}$ is optimized to be around $200-300 \mathrm{~nm}$, making sure that the $\mathrm{SiO} 2$ layer can sustain longer etching time to completely remove the residual layer. The insertion of a $\mathrm{SiO}_{2}$ layer between the Cytop layer and the resist is an important modification in the NIL process. It not only ensures the successful spin-coating of SU-8 resist, but also protects the Cytop layer from being etched. The $\mathrm{SiO}_{2}$ layer left on top of Cytop can be removed easily by hydrofluoric acid. 


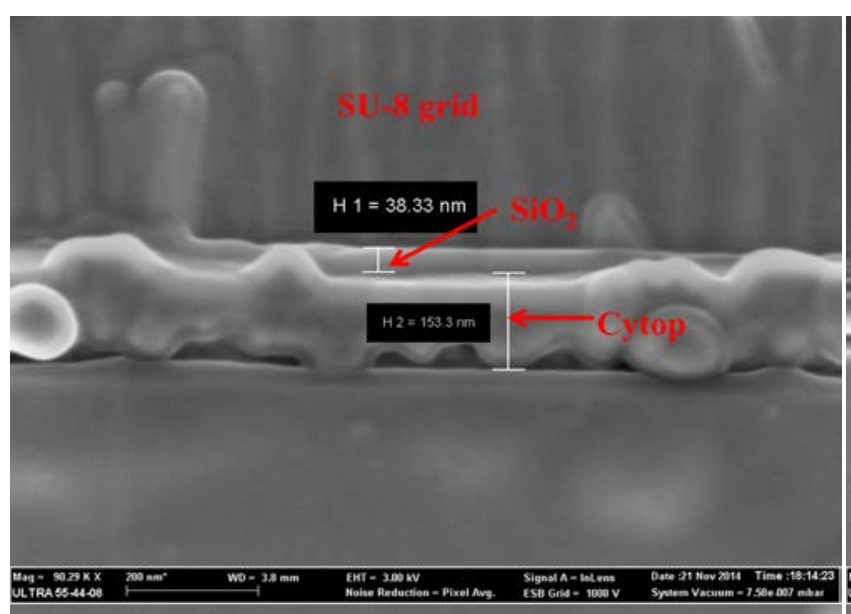

(a)

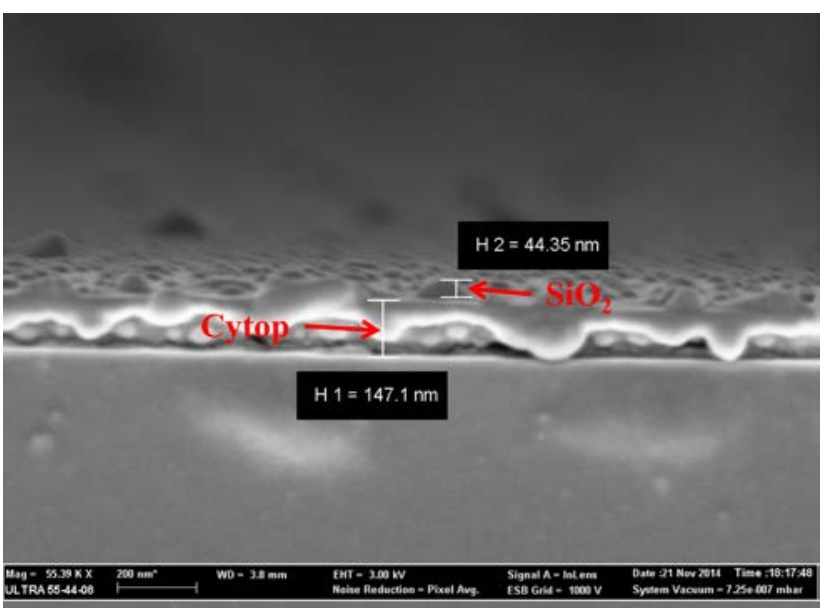

(b)

Figure 3. Q. T. Di (a) SEM images acquired after removing the residual SU-8 layer by RIE; and (b) the residual SU-8 layer is completely removed while the $\mathrm{SiO}_{2}$ layer between SU-8 grids still remains.

Rectangle pixels, $100 \mu \mathrm{m}$ in width, $300 \mu \mathrm{m}$ in length and $13 \mu \mathrm{m}$ in depth are successfully fabricated, as shown in Fig. 4. It is observed that there are few defects existing in the imprinted pixels over an area of $0.35 \times 0.35 \mathrm{~mm}^{2}$. The above results clearly demonstrate that the mold pattern has been successfully transferred into the SU-8 layer and the EWD pixels can be successfully manufactured by NIL.

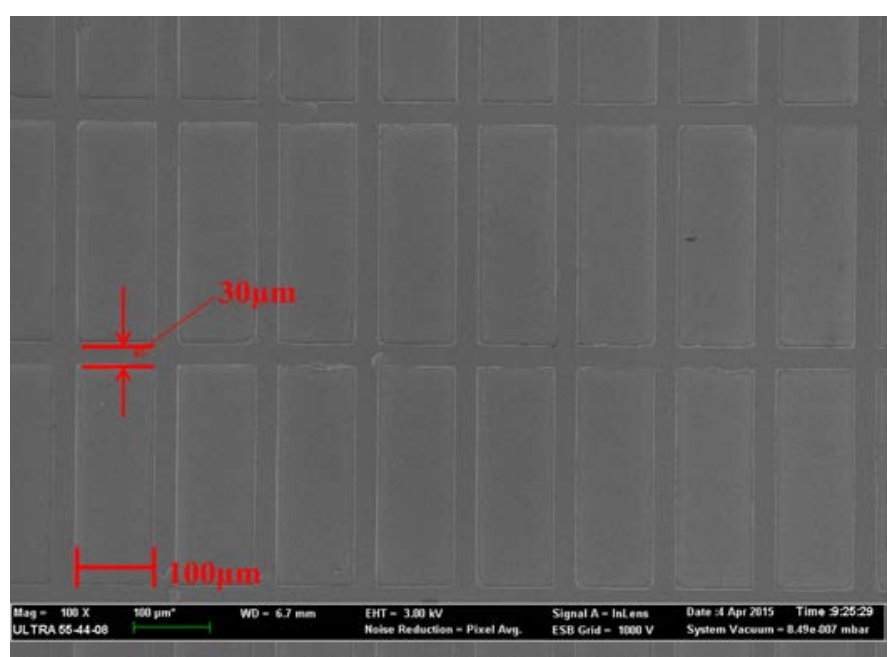

Figure 4. Q. T. Di SEM image of EWD pixels fabricated by NIL.

Fig. 5 shows a demonstration of the EWD. As shown in Fig. 5 (a), the red oil confined in the SU8 grids naturally forms a continuous film in each pixel. When a voltage is applied between the top and bottom electrodes, the oil film contracts and the underlying Cytop surface exposes, as exhibited in Fig 5 (b). However, not all the pixels work. This is because the thickness of the oil film in different pixels are not identical. It is still a challenge to fill all the pixels with the oil to the grid height. Further optimizations are required to improve the uniformity of EWD pixels. 


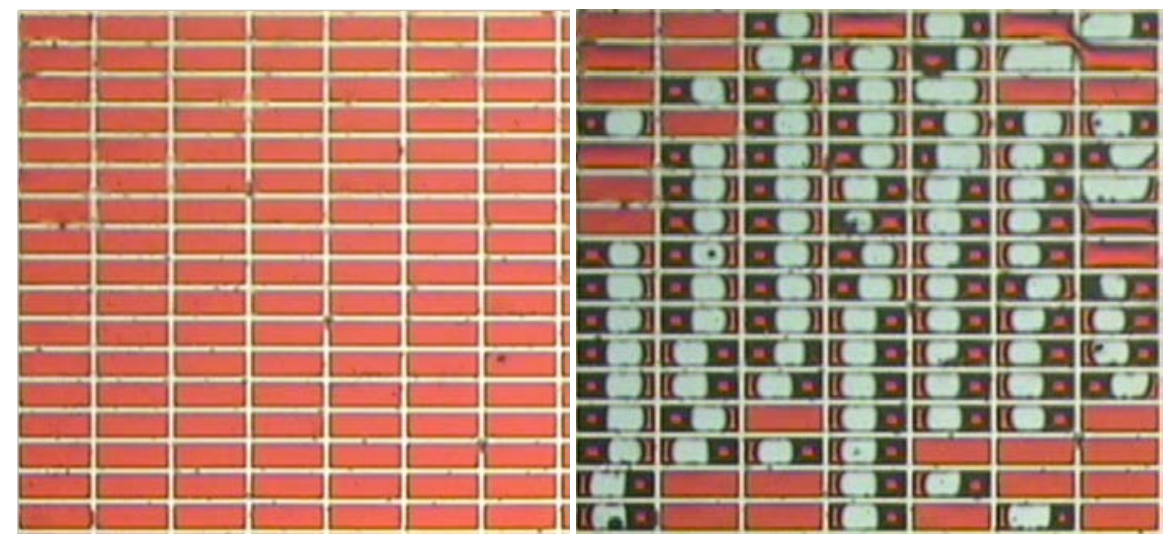

(a) OFF state

(b)ON state

Figure 5. Q. T. Di Result of EWD pixels test showing a continuous oil film in each pixel with no voltage applied (a) and the contraction of oil with voltage applied (b).

\section{Conclusions}

We successfully fabricated EWD pixels using NIL instead of conventional photolithography. By inserting a $\mathrm{SiO}_{2}$ layer deposited by PECVD, the spin-coating of SU-8 resist on the hydrophilic Cytop surface is ensured and this $\mathrm{SiO}_{2}$ also serves as a barrier to protect overetching the Cytop surface during the removal of the residual layer. Compared with the conventional lithography approach, NIL is inherently cost- and time- saving. The result of EWD test demonstrates the feasibility of NIL in EWD fabrication although further optimizations are required.

\section{Acknowledgments}

The authors thank Professor H. X. Ge for providing access to his imprint facilities and also for his valuable technical assistance.

\section{References}

[1] Hayes R A and Fenestrate B J: 'Video-speed electronic paper based on electro wetting', Nature, 2003, 425, 383-5.

[2] Mugele F and Baret J : 'Electrowetting: from basics to Applications', J. Phys-Condens Matter,2005, 17, R705-74.

[3] Y. Lao and J. Heikenfeld: 'Ultra-High Transmission Electrowetting Displays Enabled by Integrated Reflectors', Journal of Display Technology, 2008, 4, 120-122.

[4] K Zhou and J Hearkened: 'A full description of a simple and scalable fabrication process for electro wetting displays’, J. Micromesh. Microeng, 2009, 19065029.

[5] Elizabeth A. Costner and Michael W. Lin: 'Nan imprint Lithography Materials Development for Semiconductor Device Fabrication', Annual Review of Material Research, 2009, 39, 155180.

[6] L. Jay Guo: 'Nanoimprint Lithography: Methods and Material Requirements', Adv. Mater. 2007, 19 , 495-513.

[7] Helmut Schift: 'Nanoimprint lithography: An old story in modern times? A review', Journal of Vacuum Science \& Technology B, 2008, 26, 456-480.

[8] Sung-Won Youn: 'Microstructuring of SU-8 photoresist by UV-assisted thermal imprinting with non-transparent mold’, Microelectronic Engineering, 2008, 85,1924-1931 
[9] Kwang-Seop Kim and Jae-Hyun Kim: 'Tribology issues in nanoimprint lithography', Journal of Mechanical Science and Technology, 2010, 24, 5 12.

[10]Chih-Wei Wu and Yung-Kang Shen: 'Anti-adhesive effects of diverse self-assembled monolayers in nanoimprint lithography', Sensors and Actuators A, 2007, 139, 145-151.

[11]Xudi wang and Yifang Chen: 'High density patterns fabricated in SU-8 by UV curing nanoimprint', Microelectronic Engineering, 2007, 84, 872-876.

[12]Kanghun Moon and Banglim Choi: 'Novel hybrid mask mold for combined nanoimprint', Microelectronic Engineering, 2006, 83, 889-892.

[13]G. Hong and A.S. Holmes: 'SU8 resist plasma etching and its optimistion', Microsystem Technologies, 2004, 10, 357-359. 